\title{
Photoreceptor spectral sensitivities of the Small White butterfly Pieris rapae crucivora interpreted with optical modeling
}

\author{
Doekele G. Stavenga · Kentaro Arikawa
}

Received: 18 November 2010/Revised: 23 December 2010/Accepted: 25 December 2010/Published online: 13 January 2011 (C) The Author(s) 2011. This article is published with open access at Springerlink.com

\begin{abstract}
The compound eye of the Small White butterfly, Pieris rapae crucivora, has four classes of visual pigments, with peak absorption in the ultraviolet, violet, blue and green, but electrophysiological recordings yielded eight photoreceptors classes: an ultraviolet, violet, blue, double-peaked blue, green, blue-suppressed-green, pale-red and deep-red class. These photoreceptor classes were identified in three types of ommatidia, distinguishable by the different eye shine spectra and fluorescence; the latter only being present in the eyes of males. We present here two slightly different optical models that incorporate the various visual pigments, the light-filtering actions of the fluorescent, pale-red and deep-red screening pigment, located inside or adjacent to the rhabdom, and the reflectance spectrum of the tapetum that abuts the rhabdom proximally. The models serve to explain the photoreceptor spectral sensitivities as well as the eye shine.
\end{abstract}

Keywords Rhabdom - Tapetum - Screening pigment . Fluorescent pigment · Eye shine

\section{Introduction}

The compound eyes of the Small White butterfly, Pieris rapae crucivora, are composed of three types of ommatidia (Wakakuwa et al. 2007), the anatomy of which are known

D. G. Stavenga $(\bowtie)$

Department of Neurobiophysics, University of Groningen,

9747 AG Groningen, The Netherlands

e-mail: D.G.Stavenga@rug.nl

K. Arikawa

School of Advanced Sciences, The Graduate University for Advanced Studies (SOKENDAI), Hayama 240-0193, Japan in precise detail (Qiu et al. 2002). The ommatidial types are distinguished by the shape of the rhabdom; for example, the rhabdom cross-section in type I, II and III ommatidia is trapezoidal, square, and rectangular, respectively. The different rhabdom shapes are due to the different amounts of rhabdomeric microvilli that the individual photoreceptors contribute to the rhabdom. All ommatidia contain nine photoreceptors, R1-9, which are divided into three groups, according to the position of their rhabdomeres. The rhabdomeres of photoreceptors R1-4, R5-8, and R9 form the distal, proximal, and basal parts of the rhabdom, respectively. The rhabdoms in the main, fronto-ventral part of the compound eye are surrounded by four clusters of pale-red or deep-red pigment, which determine the colour of the eye shine observable with an epi-illumination light microscope. The eye shine, which is due to a tapetum that abuts the rhabdom at its proximal end, is pale-red in type I and III ommatidia, where the peri-rhabdomal pigment is pale-red, and the eye shine is deep-red in type II ommatidia, which contain the deep-red pigment. Furthermore, the type II ommatidia of male eyes fluoresce under excitation with violet light.

Extensive electrophysiological recordings of the photoreceptors, combined with optical and anatomical methods of identifying the location of the recorded cells, demonstrated that anatomically identical photoreceptors located in different ommatidia can have dissimilar spectral sensitivities. Parallel in situ hybridization studies revealed that this was due to the expression of different visual pigments or to the filtering by different screening pigments. Pieris rapae crucivora utilizes four rhodopsins, $\operatorname{PrUV}, \operatorname{PrV}, \operatorname{PrB}$, and PrL (Table 1), absorbing maximally in the ultraviolet, violet, blue and green-yellow wavelength range, respectively (Wakakuwa et al. 2004, 2007; Arikawa et al. 2005). However, intracellular recordings identified no less than 
eight different spectral classes of photoreceptors (Qiu and Arikawa 2003a; Wakakuwa et al. 2004, 2007; Stavenga and Arikawa 2006). These studies provided convincing evidence that various spectral filters, located inside and/or adjacent to the rhabdom, cause a diversification of the spectral sensitivities of the photoreceptors. So far a comprehensive description of the Pieris ommatidia, taking into account all components that shape the photoreceptor sensitivities has not been developed. Here, we present two slightly different optical models of the three ommatidial types of $P$. rapae crucivora describing the spectral sensitivities of the photoreceptors. The models provide insight into how the sensitivity spectra are shaped by the absorbance spectra of the visual and screening pigments and the tapetal reflectance, and perhaps more importantly, they allow a quantitative estimate of the absolute light sensitivities and the loss in sensitivity due to the optical filters.

\section{Materials and methods}

Ommatidial types, photoreceptor spectral sensitivities, and visual pigments

Table 1 summarizes the characteristics of the three ommatidial types of Pieris rapae crucivora, and specifies their photoreceptors, the visual and screening pigments, and the eye shine. Figure 1 presents the spectral sensitivities measured by intracellular recordings (Qiu and

Table 1 The three ommatidial types of Pieris rapae crucivora

\begin{tabular}{|c|c|c|c|c|c|c|c|c|}
\hline $\begin{array}{l}\text { Ommatidial } \\
\text { type }\end{array}$ & \multicolumn{2}{|l|}{ I } & \multicolumn{2}{|l|}{ IIf } & \multicolumn{2}{|l|}{ IIm } & \multicolumn{2}{|l|}{ III } \\
\hline $\begin{array}{l}\text { Rhabdom } \\
\text { shape }\end{array}$ & \multicolumn{2}{|c|}{ Trapezoid } & \multicolumn{2}{|c|}{ Square } & \multicolumn{2}{|c|}{ Square } & \multicolumn{2}{|c|}{ Rectangula } \\
\hline $\begin{array}{l}\text { Pigment/eye } \\
\text { shine }\end{array}$ & \multicolumn{2}{|c|}{ Pale-red } & \multicolumn{2}{|c|}{ Deep-red } & \multicolumn{2}{|c|}{ Deep-red } & \multicolumn{2}{|c|}{ Pale-red } \\
\hline Sex & \multicolumn{2}{|c|}{$\begin{array}{c}\text { Female/ } \\
\text { male }\end{array}$} & \multicolumn{2}{|c|}{ Female } & \multicolumn{2}{|c|}{ Male } & \multicolumn{2}{|c|}{$\begin{array}{c}\text { Female/ } \\
\text { male }\end{array}$} \\
\hline $\begin{array}{c}\text { Fluorescing } \\
\text { pigment }\end{array}$ & \multicolumn{2}{|l|}{ No } & \multicolumn{2}{|l|}{ No } & \multicolumn{2}{|l|}{ Yes } & \multicolumn{2}{|l|}{ No } \\
\hline Photoreceptor & $S(\lambda)$ & Opsin & $\mathbf{S}(\lambda)$ & Opsin & $\mathrm{S}(\lambda)$ & Opsin & $\mathrm{S}(\lambda)$ & Opsin \\
\hline $\mathrm{R} 1$ & $\mathrm{UV}^{\mathrm{a}}$ & PrUV & $\mathrm{V}$ & PrV & $\mathrm{dB}$ & PrV & UV & PrUV \\
\hline $\mathrm{R} 2$ & $\mathrm{~B}^{\mathrm{a}}$ & PrB & $\mathrm{V}$ & $\operatorname{PrV}$ & $\mathrm{dB}$ & PrV & UV & PrUV \\
\hline $\mathrm{R} 3,4$ & G & PrL & G & PrL & $\mathrm{dG}$ & PrL & G & PrL \\
\hline $\mathrm{R} 5-8$ & $\mathrm{PR}$ & PrL & $\mathrm{DR}$ & PrL & DR & PrL & PR & PrL \\
\hline R9 & PR? & PrL? & DR? & PrL? & DR? & PrL? & $\mathrm{PR} ?$ & PrL? \\
\hline
\end{tabular}

$S(\lambda)$ gives the name of the photoreceptor class, as derived from the wavelength range of the spectral sensitivity, $U V$ ultraviolet, $V$ violet, $d B$ double-peaked blue, $B$ blue, $G$ green, $d G$ blue-suppressed green, $P R$ pale-red, $D R$ deep-red (Wakakuwa et al. 2007)

a The anatomically defined photoreceptors R1 and R2 either contain the UV and B rhodopsin or the B and UV rhodopsin
Arikawa 2003a, b; Wakakuwa et al. 2004; Arikawa et al. 2005). The spectra of the ultraviolet (UV), violet (V), blue $(B)$, and green $(\mathrm{G})$ and blue-suppressed green $(\mathrm{dG})$ receptors were previously fitted with rhodopsin spectra peaking at 360, 425, 453 and $563 \mathrm{~nm}$, respectively (Fig. 1a), but recent expression studies indicated that the rhodopsins of the violet and blue receptors were better described by R420 and R450 (that is, they peak at 420 and $450 \mathrm{~nm}$; Wakakuwa et al. 2010), and in the course of the present modeling work we concluded that the ultraviolet as well as the green rhodopsin are more closely described by R350 and R560. For the R350 rhodopsin spectrum we used the template of Stavenga et al. (1993), because this template fits the UVphotoreceptor spectra better than the template of Govardovskii et al. (2000), which was used for the spectra of R420, R450, and R560 (see Stavenga 2010). In the measured spectral data presented in Fig. 1b, those of the double-peaked blue $(\mathrm{dB})$, pale-red $(\mathrm{PR})$ and deep-red (DR) receptors deviate severely from template spectra. The $\mathrm{dB}$ receptors are the $\mathrm{R} 1$ and $\mathrm{R} 2$ photoreceptors of type II
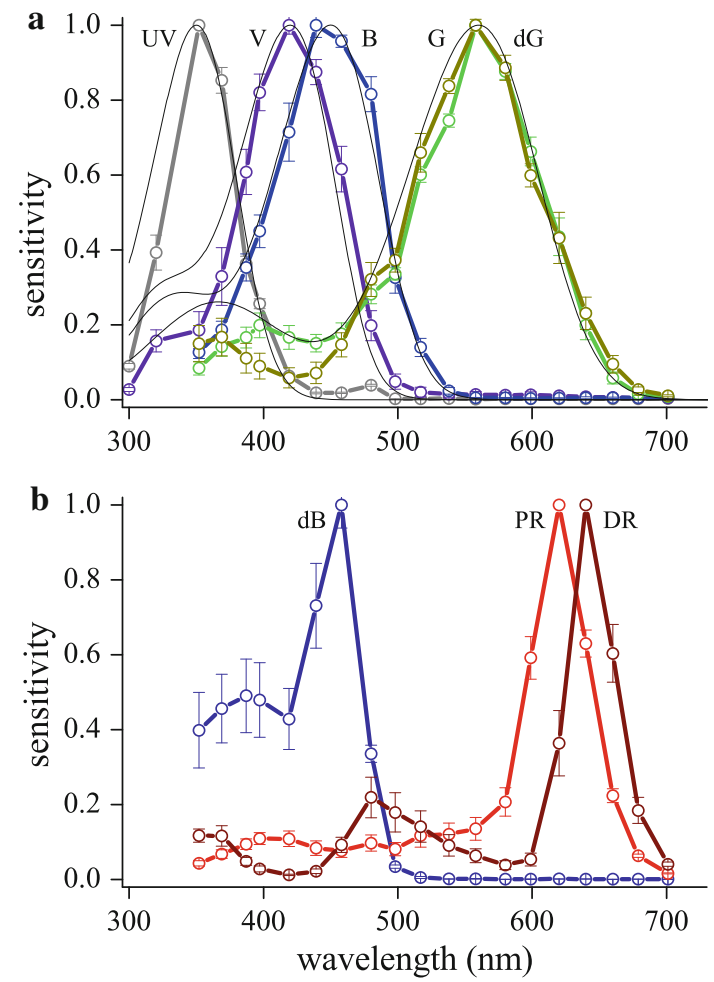

Fig. 1 Spectral sensitivities of photoreceptors of the Small White butterfly, Pieris rapae crucivora (from Qiu and Arikawa 2003a, b; Wakakuwa et al. 2004; Arikawa et al. 2005). a Spectral sensitivities of ultraviolet $(U V)$, violet $(V)$, blue $(B)$ and green-sensitive photoreceptors ( $G$ green, $d G$ blue-suppressed green) shown together with more or less approximating, template-predicted visual pigment spectra, with peak wavelengths $350,420,450$ and $560 \mathrm{~nm}$, respectively (thin black lines). b Spectral sensitivities of the double-peaked blue receptor $(d B)$, and the pale-red $(P R)$ and deep-red $(D R)$ photoreceptors that strongly deviate from template-predicted spectra 
ommatidia of males, which we call here ommatidial type IIm to distinguish it from the female type IIf (Table 1). The R1 and R2 photoreceptors of both type IIm and IIf ommatidia contain the same rhodopsin, R420. In type IIf the $\mathrm{R} 1$ and $\mathrm{R} 2$ are therefore $\mathrm{V}$ receptors, but because type IIm ommatidia contain a violet-absorbing, fluorescent pigment the spectra of the $\mathrm{R} 1$ and $\mathrm{R} 2$ are modified into $\mathrm{dB}$ receptors (review Wakakuwa et al. 2007). In situ hybridization studies demonstrated that the R3-8 photoreceptors of all ommatidial types contain the same mRNA encoding the opsin of PrL rhodopsin, R560. The R3,4 are therefore G receptors, but due to filtering by the pale-red screening pigments in ommatidial types I and III, the R5-8 are modified into $\mathrm{PR}$ receptors, and due to filtering by the deep-red screening pigments in ommatidial types IIm and IIf their R5-8 become DR receptors (Wakakuwa et al. 2004). The suppressed sensitivity in the blue wavelength region of the $\mathrm{R} 3,4 \mathrm{dG}$ receptors in type IIm ommatidia is due to the violet-absorbing, fluorescing pigment.

A simple model for the butterfly ommatidium

The diagram of Fig. 2 (left) presents the optical components of a schematic ommatidium of $P$. rapae crucivora, i.e., the facet lens, crystalline cone, fused rhabdom and tapetum. The rhabdom consists of a distal, proximal and basal part, with the rhabdomeres of photoreceptors R1-4, R5-8 and R9, respectively. The rhabdom is surrounded by red screening pigment over a restricted distance. Incident light is focused by the facet lens and crystalline cone into the rhabdom, where it then propagates in waveguide modes (the profiles of the first and second mode are sketched in Fig. 2), until the light is absorbed by the visual pigment of the photoreceptors or by the screening pigment surrounding the rhabdom. A fraction of the propagating light however escapes absorption and reaches the tracheolar tapetum, which abuts the rhabdom. The tapetum acts as an interference reflector, and thus part of the light travels in the reverse direction and can leave the eye as the eye shine.

The light fraction absorbed from the incident light flux by the individual photoreceptors can be calculated when their spectral as well as anatomical properties are known. On the basis of the anatomical details of the ommatidial types reported by Qiu et al. (2002) we have made the following assumptions. The rhabdom tip is located at $z=0 \mu \mathrm{m}$; the transition of the distal to proximal layer of the rhabdom in all three ommatidial types occurs at $z=220 \mu \mathrm{m}$; the transition of the proximal to basal layer occurs at $z=390 \mu \mathrm{m}$; and the rhabdom ends at $z=400 \mu \mathrm{m}$. The pale-red pigment in ommatidial types I and III extends from $z=100$ to $280 \mu \mathrm{m}$, and the deep-red pigment of ommatidial type IIm and IIf extends from $z=60$ to $280 \mu \mathrm{m}$. The fluorescent pigment of type IIm

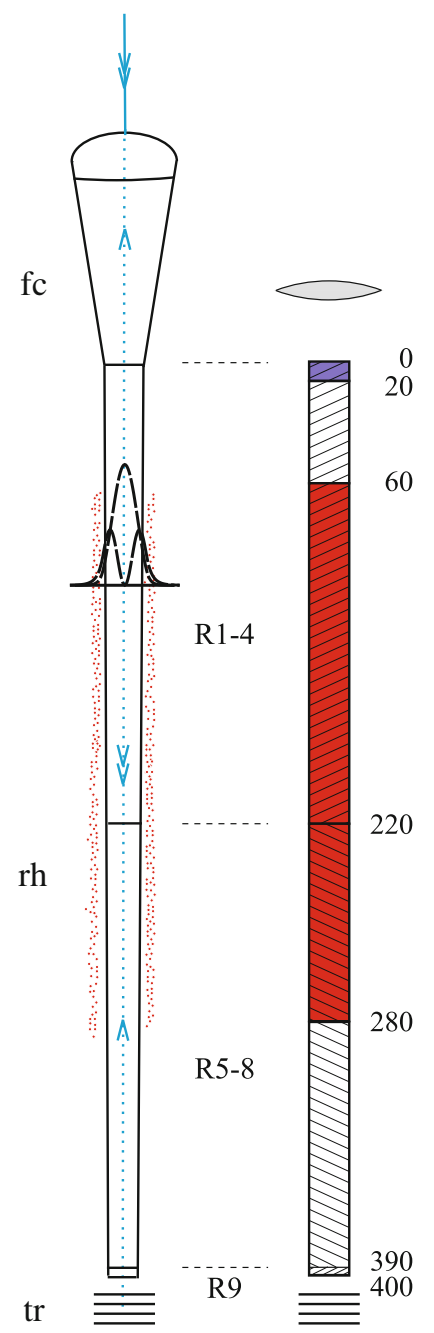

Fig. 2 Diagrams of an ommatidium of $P$. rapae crucivora. Light enters the facet lens and crystalline cone $(f c)$ and then is focused into the rhabdom $(r h)$. The rhabdom consists of rhabdomeres, distally of the photoreceptors $R 1-4$, proximally of photoreceptors $R 5-8$, and basally of photoreceptor $R$. The rhabdom is partly surrounded by red pigment. Proximal to the rhabdom a tracheole exists, which acts as a tapetum. Left light propagates in the tapering rhabdom in waveguide modes; the profiles of the two lowest order modes are shown. Right simple compartment model of the type IIm ommatidium, of the male butterfly, with the R1-4 rhabdomeres taking up the distal $220 \mu \mathrm{m}$ of the rhabdom, the R5-8 rhabdomeres the proximal $170 \mu \mathrm{m}$, and the R9 rhabdomere the basal $10 \mu \mathrm{m}$. The top distal $20 \mu \mathrm{m}$ of the rhabdom contains a fluorescent, violet-absorbing pigment, and a deep-red pigment pigment is present from 60 to $280 \mu \mathrm{m}$. The lens shape in front of the rhabdom indicates the non-perfectly transparent dioptrical system

ommatidia is present only in the most distal $20 \mu \mathrm{m}$ of the rhabdom. The rhabdomeres occupy unequal parts of the rhabdom cross-section, depending on the ommatidial types. Table 2 gives the relative size of the rhabdomeres, or their occupancy ratio. Whereas the occupancy ratio of the rhabdomeres of R1-4 in the distal layer distinctly differs between the three ommatidial types, the rhabdomere sizes 
Table 2 Occupancy ratio $\rho_{i}$ of rhabdomeres Ri ( $i=1-9$; columns) in the distal (R1-4), proximal (R5-8), and basal (R9) layer of ommatidia I, II, and III (rows)

\begin{tabular}{|c|c|c|c|c|c|c|}
\hline & \multicolumn{4}{|c|}{ Distal } & \multirow{2}{*}{$\begin{array}{l}\text { Proximal } \\
\text { R5-8 }\end{array}$} & \multirow{2}{*}{$\begin{array}{l}\text { Basal } \\
\text { R9 }\end{array}$} \\
\hline & $\mathrm{R} 1$ & $\mathrm{R} 2$ & R3 & $\mathrm{R} 4$ & & \\
\hline I & 0.4 & 0.3 & 0.15 & 0.15 & 0.25 & 1 \\
\hline IIf, IIm & 0.3 & 0.3 & 0.2 & 0.2 & 0.25 & 1 \\
\hline III & 0.35 & 0.35 & 0.15 & 0.15 & 0.25 & 1 \\
\hline
\end{tabular}

of R5-8 in the proximal layer are equal, so there each rhabdomere occupies a fraction $\rho=0.25$ of the crosssection, and the rhabdomere of R9 in the basal layer occupies the full rhabdom cross-section $(\rho=1)$.

We implemented the above values in a simple model of the ommatidia where the wave-optical properties of the dioptrical system and rhabdom were neglected. Figure 2 (right) shows ommatidial type IIm in this case. We thus assumed that the light fully propagates inside the rhabdom and that, like the visual pigments, the red screening pigments as well as the male fluorescent pigment exert their filtering actions as if they were within the rhabdom. The transmittance $[T(\lambda)]$ of a rhabdom layer with thickness $\Delta z$ then is given by

$T(\lambda)=\exp [-\kappa(\lambda) \Delta z]$

with

$\kappa(\lambda)=\kappa_{\mathrm{v}}(\lambda)+\kappa_{\mathrm{s}}(\lambda)$

where $\kappa_{\mathrm{v}}$ is the absorbance coefficient due to absorption by the visual pigments given by

$\kappa_{\mathrm{v}}=\kappa_{\mathrm{v}, \max }\left\{\sum \alpha_{i}(\lambda) \rho_{i}\right\}$

with $\kappa_{\mathrm{v}, \max }$ the peak absorbance coefficient, $\alpha_{i}(i=1-9)$ the normalized absorbance spectrum of the visual pigment in receptor $\mathrm{R} i$ (that is, one of the four visual pigments R350, R420, R450, R560), and

$\kappa_{\mathrm{s}}=\kappa_{\mathrm{s}, \max } \alpha_{\mathrm{s}}(\lambda)$

with $\kappa_{\mathrm{s}, \max }$ the peak absorbance coefficient and $\alpha_{\mathrm{s}}$ the normalized absorbance spectrum of the screening pigment (Fig. 3a, b). The light flux at location $z, I(z, \lambda)$, then changes into $I(z+\Delta z, \lambda)$, the lightflux at location $z+\Delta z$, by

$I(z+\Delta z, \lambda)=T(\lambda) I(z, \lambda)$.

The light flux absorbed by the visual pigment of photoreceptor $\mathrm{R} i$ in the considered compartment with thickness $\Delta z$ is given by

$$
\Delta A_{i}(\lambda)=[I(z, \lambda)-I(z+\Delta z, \lambda)] \kappa_{v, \max } \alpha_{i}(\lambda) \rho_{i} / \kappa(\lambda) .
$$

Summing the absorbed light fraction of the individual photoreceptors over the different rhabdom compartments and dividing that by the incident light, $I_{\mathrm{i}}(\lambda)$, then yields the photoreceptor's absorptance spectrum. Normalizing the latter yields the spectral sensitivity. The summation procedure has to be performed for the total pathway, from the distal rhabdom tip to the tapetum and back, taking into account the reflectance spectrum of the tapetum. We have deduced the tapetal reflectance spectra by using the experimentally obtained average eye shine spectra (Fig. 3 of Qiu et al. 2002). Formally, if $T_{\mathrm{C}}(\lambda)$ is the transmittance spectrum of the corneal facet lens and crystalline cone, the light flux spectrum at $z=0$ is $I_{0}(\lambda)=T_{\mathrm{C}}(\lambda) I_{\mathrm{i}}(\lambda)$; if the light flux reaching the tapetum, at $400 \mu \mathrm{m}$, is $I_{400}(\lambda)$, then the transmittance spectrum of the rhabdom is $T_{\mathrm{r}}(\lambda)=I_{400}(\lambda) / I_{0}(\lambda)$. With the tapetal mirror given by $M(\lambda)$, the measured eye shine spectrum equals $E(\lambda)=$ $T_{\mathrm{C}}(\lambda) T_{\mathrm{r}}(\lambda) M(\lambda) T_{\mathrm{r}}(\lambda) T_{\mathrm{C}}(\lambda)=T_{\mathrm{C}}(\lambda)^{2} T_{\mathrm{r}}(\lambda)^{2} M(\lambda), \quad$ or, $\quad$ the tapetal mirror spectrum can then be deduced from the eye shine spectrum: $M(\lambda)=E(\lambda) T_{\mathrm{C}}(\lambda)^{-2} T_{\mathrm{r}}(\lambda)^{-2}$. However, it is extremely difficult to assess the absolute value of the eye shine reflectance, whereas the shape of the eye shine reflectance spectrum can be readily measured. The tapetal reflectance spectra, $M(\lambda)$, are therefore presented normalized (Fig. 3a, b). Furthermore, the eye shine measurements always contain some background due to reflections on the corneal facet lenses and scattering by other retinal tissues. The calculations showed that the light flux in the return pathway is very minor for $\lambda<\lambda_{\min } \approx 570 \mathrm{~nm}$ in type I and III ommatidia, and for $\lambda<\lambda_{\min } \approx 620 \mathrm{~nm}$ in type II ommatidia, and therefore the spectral sensitivity calculations are virtually insensitive to the value of the tapetal reflectance in these wavelength ranges. We therefore have taken the tapetal reflectance to be equal to 1 at $\lambda<\lambda_{\min }$.

The wave-optical model

The simple model neglects wave-optical effects, which is of course an oversimplification. Unfortunately, notwithstanding important contributions (van Hateren and Nilsson 1987; Nilsson et al. 1988), the optics of the butterfly ommatidia is not sufficiently known to allow a fully adequate analysis of the passage of light via the facet lens and the crystalline cone and the excitation of waveguide modes upon the entering of the light flux into the rhabdom. Previously a detailed treatment has been given for the optics of fly eyes (Stavenga 2003), where the cone can be assumed to be homogeneous, but this is certainly not the case for butterflies (Nilsson et al. 1988). Therefore, we have applied a heuristic approach by assuming that the optics of the butterfly eye behaves similarly to that of the fly. We took a facet lens diameter of $22 \mu \mathrm{m}$ (Qiu et al. 2002) with an Fnumber 2.4. The rhabdom was assumed to taper linearly, from its tip at $z=0 \mu \mathrm{m}$ to the basal end at $z=400 \mu \mathrm{m}$, from a tip diameter $2.0 \mu \mathrm{m}$ to a basal diameter $1.5 \mu \mathrm{m}$ (Qiu et al. 2002). The excitation of allowed waveguide modes 
Fig. 3 Simple ommatidium model calculations of the light flux, photoreceptor absorptances and spectral sensitivities. Left column type I (solid curves) and III ommatidia (dashed curves), right column type IIf (solid curves) and IIm ommatidia (dashed curves).

a, b Absorbance spectra, normalized to the peak in the visible wavelength range, of the pale-red pigment (in a) and deep-red screening (scr pigm) pigments (in b); the normalized reflectance spectra of the tapeta (tapetum) used in the calculations; the experimentally measured eye shine spectra (eye shine); and the fluorescent pigment of male eyes ( $f$ pigm). a inset light-microscopical section of a compound eye of Pieris rapae crucivora (from Arikawa and Stavenga 1997) showing the clusters of pale-red (dashed circle) and deep-red pigment (solid circle) that surround the rhabdoms.

c, d Light flux at rhabdom levels $z=0,20,100(\mathbf{c})$ or $60(\mathbf{d})$, 220 , and $390 \mu \mathrm{m}$ for forward propagating light, and at levels 400 and $0 \mu \mathrm{m}$ for light traveling the reverse (r) way.

e, f Absorptance spectra for photoreceptor R1, R2, R3,4, R5-8, and R9. g, h Spectral sensitivities obtained by normalizing the photoreceptor absorptance spectra are compared with the corresponding experimental spectral sensitivities (taken from Fig. 1) $\mathbf{a}$

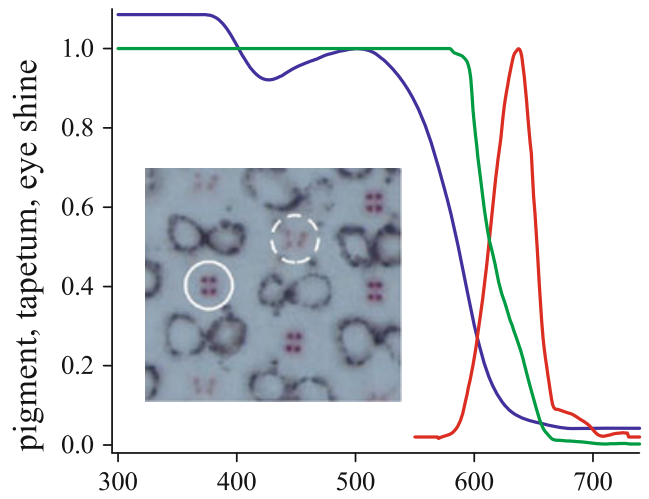

c

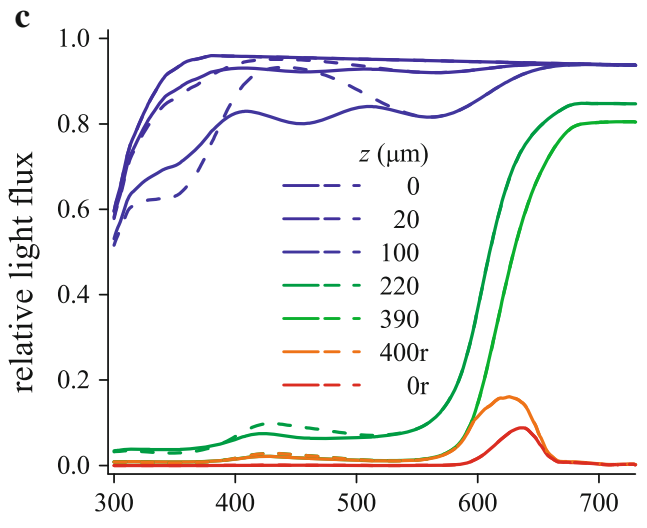

e

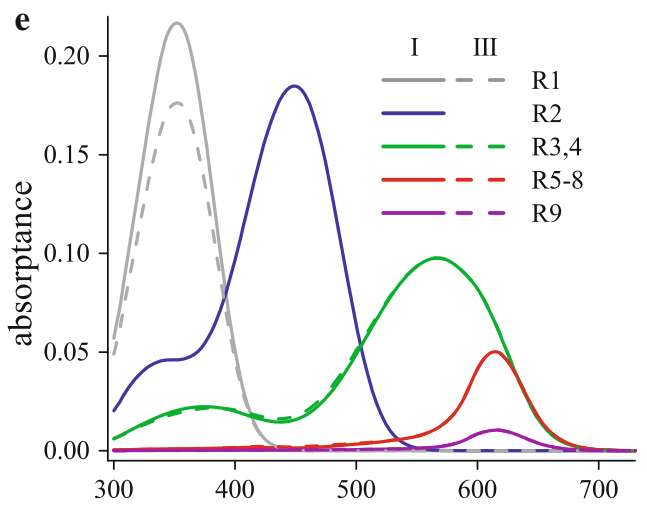

g

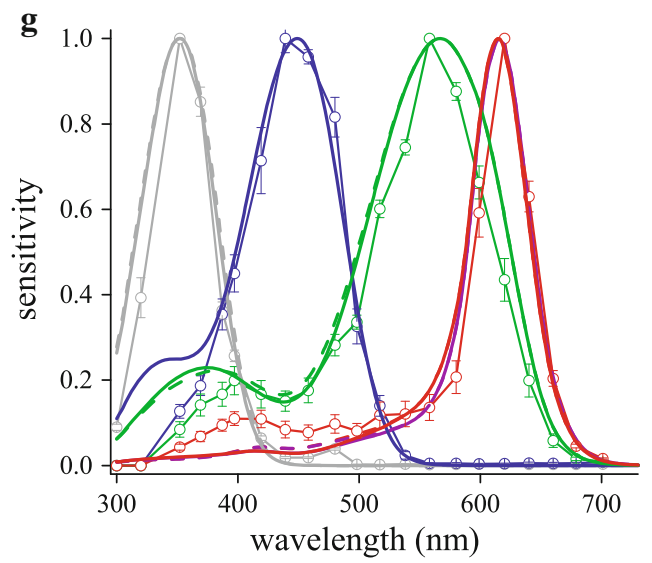

b

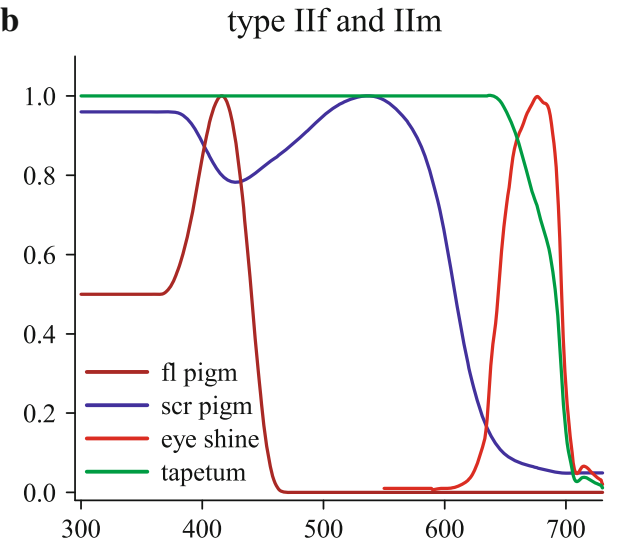

d
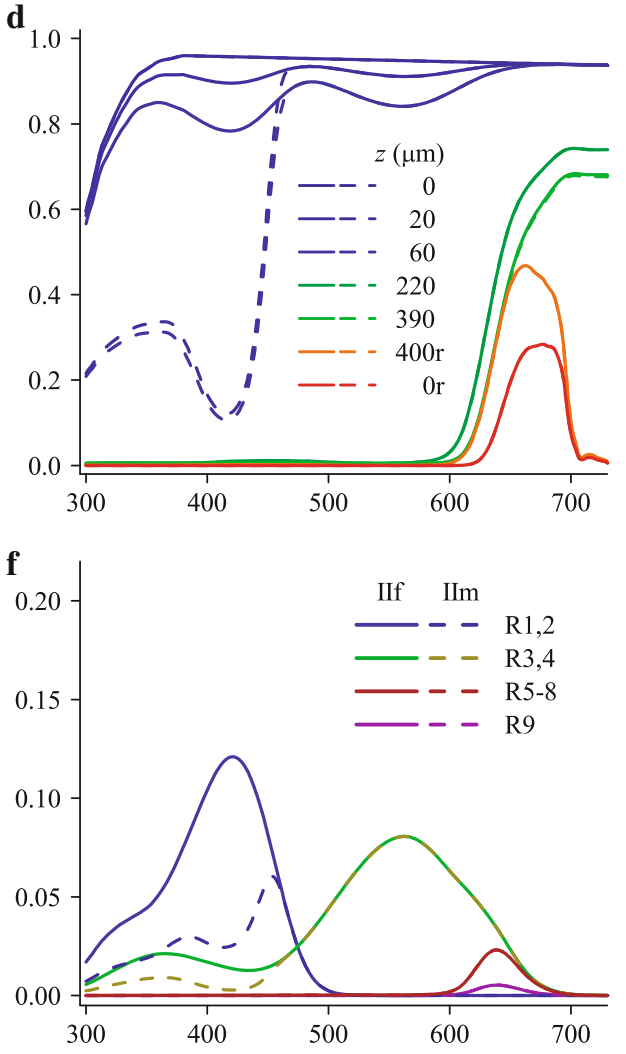

h

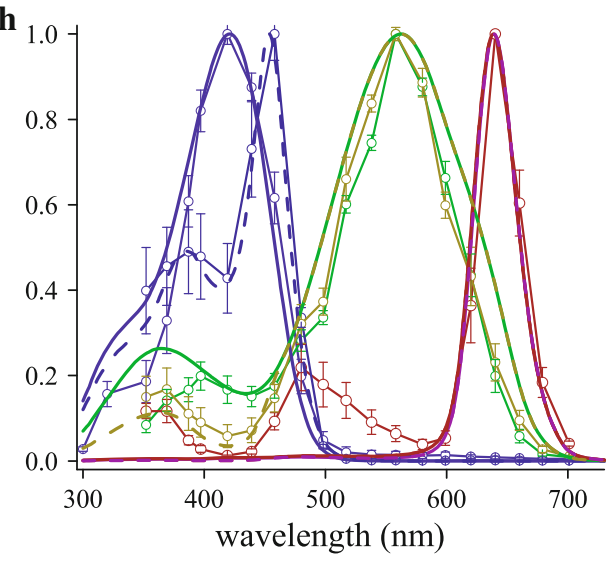


with mode number $p$ by an extended light source with a unit irradiance $\left(1 \mathrm{~W} \mathrm{sr}^{-1} \mu \mathrm{m}^{-2}\right)$ at all wavelengths was calculated as described before (Stavenga 2004). We subsequently calculated the light absorbed by the visual pigments of the butterfly photoreceptors present in the different sections of the rhabdom using the same parameters as in the simple model. However, now for each mode $\kappa_{\mathrm{v}}(\lambda)$ was multiplied by $\eta_{p}(\lambda)$, the light fraction that propagates within the rhabdom boundary. Because the screening pigment absorbs light outside the rhabdom boundary, $\kappa_{\mathrm{s}}(\lambda)$ was multiplied by $1-\eta_{p}(\lambda)$, the light fraction that propagates outside the rhabdom boundary. The light flux and photoreceptor absorptions in each rhabdom compartment hence was calculated for each mode using $\bar{\eta}_{p}(\lambda)$, the average value of $\eta_{p}(\lambda)$ in the compartment (see Stavenga 2004). The light absorbed by the photoreceptors summed over all modes and rhabdom compartments then yielded the photoreceptors' total absorption. Normalizing the latter yielded the spectral sensitivity of the various photoreceptors.

Filtering and screening pigment absorbance spectra

The absorbance spectra of the red screening pigments that cluster near the rhabdom were measured on $10-\mu$ m-thick light-microscopical sections with a microspectrophotometer using a Zeiss Plan Neofluar $40 \times / 0.4$ objective. The ommatidia with pale-red and deep-red pigment were visually distinguished, and the absorbance spectra of about 25 ommatidia of both types were averaged and normalized at the long-wavelength peak. The spectra could not be reliably measured for wavelengths $<380 \mathrm{~nm}$, due to spectral limitations of the microspectrophotometer, and therefore the spectra are extrapolated in that wavelength range (Fig. 3a, b). The absorbance spectrum of the fluorescent pigment in type IIm ommatidia was taken to be that as derived by Arikawa et al. (2005): a lognormal function with peak wavelength $416 \mathrm{~nm}$, skewness 1.7 and halfbandwidth $60 \mathrm{~nm}$ (Fig. 3b). The spectrum was heuristically extended in the ultraviolet wavelength range by a value half the peak value (Fig. 3b). The fluorescent pigment was assumed in both the simple and wave-optics model to be fully within the rhabdom, distributed homogeneously in the top $20 \mu \mathrm{m}$.

\section{Results}

Pale-red and deep-red pigments and eye shine

Microspectrophotometrical measurements on sections of the eyes of $P$. rapae crucivora (Fig. 3a, inset) with the pale-red pigment of ommatidial type I and type III and the deep-red pigment of type II ommatidia yielded absorbance spectra with peaks at about $500 \mathrm{~nm}$ (Fig. 3a) and $535 \mathrm{~nm}$ (Fig. 3b), respectively. The measured spectra were unreliable in the ultraviolet wavelength range and, therefore, we extrapolated the absorbance into the ultraviolet wavelength range by assuming that the absorbance was constant below $370 \mathrm{~nm}$. Figure 3a, b also shows the average eye shine spectra measured with in vivo microspectrophotometry of ommatidia with pale-red and deep-red pigments (Qiu et al. 2002).

Spectral sensitivities calculated with a simple model

The red peri-rhabdomal pigments act as spectral filters for the colocalized visual pigments, and hence the sensitivity spectra of the photoreceptors in the different ommatidia will deviate from the absorbance spectra of their visual pigments. In the first part of our modeling exercise, aimed to quantitatively understand the photoreceptor spectral sensitivities, we have calculated the light flux in the rhabdom and the resulting photoreceptor sensitivity spectra with a simple model. Figure $3 \mathrm{a}, \mathrm{c}, \mathrm{e}, \mathrm{g}$ presents the spectra for ommatidial types I and III, and Fig. 3b, d, f, h shows the spectra for ommatidial types IIf and IIm. We assumed that at all wavelengths a unit light flux enters the ommatidia. Before reaching the photoreceptors the incident light first has to pass the dioptrical system, consisting of the corneal facet lens and crystalline cone. We assumed that the transmittance of the dioptrical system, $T_{\mathrm{C}}(\lambda)$, was equivalent to that of the moth Endromis (Bernhard et al. 1965). Its transmittance spectrum, $T_{\mathrm{C}}(\lambda)$, is thus identical to the relative light flux spectrum $I_{0}(\lambda)$ at $z=0 \mu \mathrm{m}$, the tip of the rhabdom (Fig. 3c, d). The spectra of Fig. 3c-h were obtained by using for all visual pigments a peak absorbance coefficient $\kappa_{\mathrm{v}, \max }=0.005 \mu^{-1}$. The values of the peak absorbance coefficients of the screening pigments were adjusted until satisfactory fits were obtained as judged by eye. We used for the pale-red and deep-red pigments peak absorbance coefficients $\kappa_{\mathrm{s}, \max }=0.02$ and $0.03 \mu \mathrm{m}^{-1}$, respectively, and we took $\kappa_{\mathrm{s} \text {,max }}=0.10 \mu^{-1}$ for the peak absorbance coefficient of the fluorescent pigment in IIm ommatidia.

With increasing depth, $z$, the light flux in the rhabdom decreases, first due to absorption by the visual pigments, but deeper down predominantly due to absorption by the red screening pigment (Fig. 3c, d). Figure 3c shows the light flux of the forward propagating beam in ommatidial types I and III at 0, 20, 100, 220, and $390 \mu \mathrm{m}$, and Fig. 3d shows the light flux in ommatidia IIm and IIf at 0, 20, 60, 220, and $390 \mathrm{~nm}$ (see Fig. 2, right). Whereas, in ommatidia I, IIf, and III the light flux at $20 \mu \mathrm{m}$ is only slightly reduced, in the case of type IIm the light flux is already considerably diminished in the (ultra)violet because of the 
distal presence of the short-wavelength absorbing, fluorescent pigment (Fig. 3d). Due to the severely filtering red pigments only red to far-red light remains near the basal level $(z=390 \mu \mathrm{m})$ in all cases. The visual pigments also reduce the light flux with increasing $z$, but their filtering actions are clearly very moderate compared to the fluorescent and the red screening pigments. Figure $3 \mathrm{c}$, d also shows the light flux spectra on the way back, at $z=400 \mu \mathrm{m}$ after reflection at the tapetum (400r), and at the corneal level after completion of the return journey $(0 r)$. The last spectrum fits well the experimentally determined eye shine spectrum of Fig. 3a, b when normalized. The details of the relative light flux spectra depend on the population of the photoreceptors. For instance, whereas in type I ommatidia photoreceptors R1 and R2 contain rhodopsins R350 and R450, in type III ommatidia both R1 and $\mathrm{R} 2$ contain R350 (Table 1). The absence of R450 in type III ommatidia causes the higher relative light flux around $450 \mathrm{~nm}$ (dashed lines in Fig. 3c) compared to type I ommatidia (solid lines in Fig. 3c).

Figure $3 \mathrm{e}, \mathrm{f}$ presents the photoreceptor absorptances, the light fractions absorbed by the different photoreceptors in the various ommatidial types. The absorptance of the R1 photoreceptors of type I ommatidia, which contain a UV rhodopsin, is slightly higher than the absorptance of the two UV receptors of type III ommatidia, first because of the larger relative occupancy of the R1 in type I ommatidia (Table 2), and secondly because in type III ommatidia both UV receptors are sharing the same light flux (lateral filtering; Fig. 3e). The R1 and R2 photoreceptors in type II ommatidia of the female (IIf) and male (IIm) contain the same violet-absorbing rhodopsin R420, but the R1 and R2 absorptance is severely reduced in the IIm ommatidia due to filtering by the distally located fluorescent pigment (Fig. 3f). The absorptances of the R3 and R4 photoreceptors in type I and III ommatidia are virtually identical and the same holds for the R5-8 and R9 photoreceptors (Fig. 3e), because the filtering effects of the different rhodopsins in the R1 and R2 photoreceptors are very minor compared to the screening pigment filters. However, the absorptance of the R3 and R4 photoreceptors in the IIm ommatidia is distinctly lower in the short wavelength range compared to the absorptance of the R3 and R4 photoreceptors in the IIf ommatidia because of the filtering effect of the fluorescent pigment.

Knowing the absorptance spectra of the individual photoreceptors, we can calculate the total light fraction absorbed by all photoreceptors, R1-9, of the different ommatidial types, i.e., the total absorptance of the various ommatidia (Fig. 4). Figure 3c, d shows that virtually all light below $600 \mathrm{~nm}$ is absorbed, and thus it follows from Fig. 4 that in all cases a major light fraction is absorbed by the screening pigments.

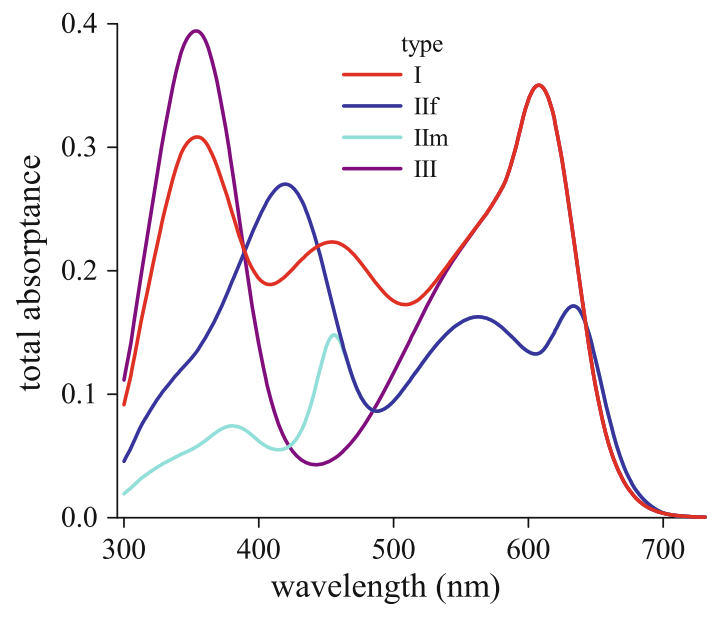

Fig. 4 Light fraction absorbed from the incident light by the set of 9 photoreceptors of the four ommatidial types

Normalization of the absorptance spectra of Fig. 3e, $\mathrm{f}$ yields the photoreceptor sensitivity spectra. Figure $3 g$, h shows the normalized spectra together with photoreceptor sensitivity spectra measured electrophysiologically (see Fig. 1). The calculated and measured sensitivity spectra match reasonably well (Fig. 3g, h), but distinct deviations are apparent. For instance, the calculated spectra for the $\mathrm{G}$ and $\mathrm{dG}$ receptors are somewhat broader than the measured spectra, the calculated spectra for the PR receptors are too low in the blue, and the DR receptors lack the distinct sensitivity hump around $480 \mathrm{~nm}$ of the measured spectra. Therefore, we speculated that these deviations could be due to the overly simplified model, and that a model which incorporates the wave-optical properties of the butterfly ommatidium might yield more accurate results.

Spectral sensitivities calculated with a wave-optics model

In the wave-optics approach we have heuristically assumed that the optics of the ommatidia of $P$. rapae crucivora behaves as if a single lens with diameter $22 \mu \mathrm{m}$ and Fnumber $F=2.4$ focuses light into a waveguide with tip diameter $2 \mu \mathrm{m}$. Figure $5 \mathrm{a}$ shows the mode power of the four permitted modes excited by an extended light source with unit irradiance $1 \mathrm{~W} \mathrm{sr}^{-1} \mu \mathrm{m}^{-2}$ at all wavelengths. The light absorption by the photoreceptors can then be calculated in a similar way to the simple model above by first calculating the light absorbed by the photoreceptors from the individual modes in each rhabdom compartment, then taking the sum of the absorbed mode fractions, and subsequently summating the absorptions over all rhabdom compartments. The visual pigments can absorb only that part of the modes which propagates inside the rhabdom boundary, $\eta_{p}$, and the complementary fraction, $1-\eta_{p}$, is 

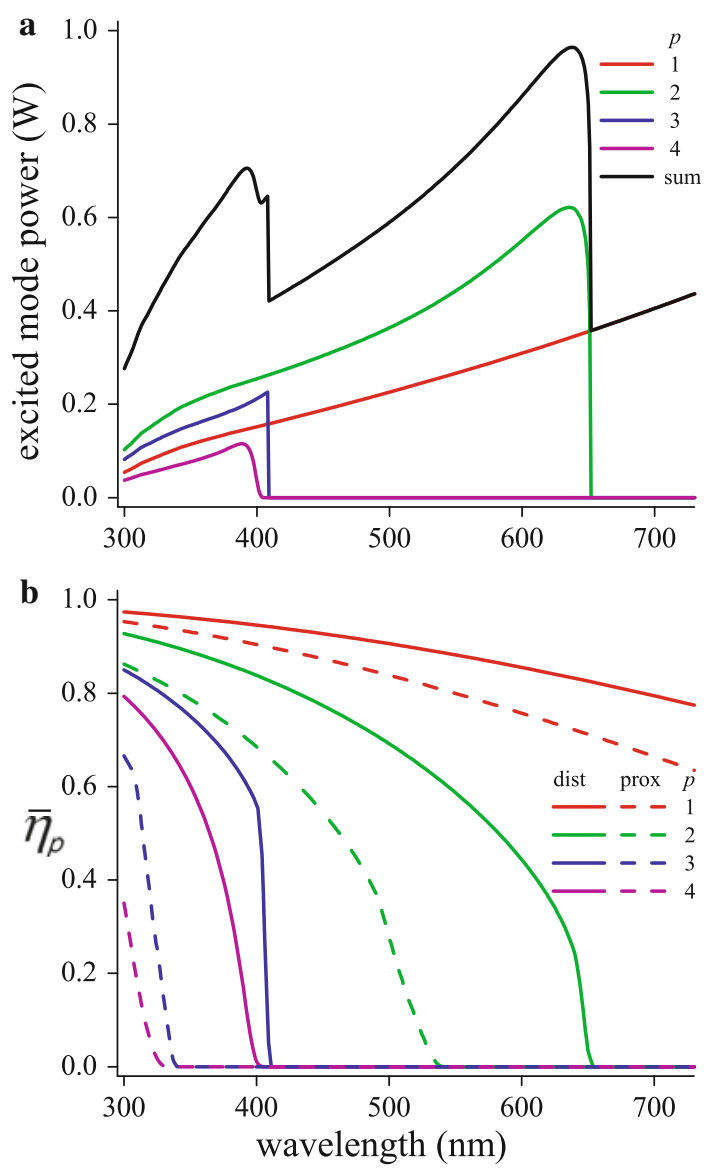

Fig. 5 Characteristics of waveguide modes in the rhabdom of Pieris rapae crucivora. a Light power delivered by an extended source entering a rhabdom with tip diameter $2 \mu \mathrm{m}$, via a facet lens with diameter $22 \mu \mathrm{m}$ and F-number $F=2.4$, exciting modes with number $p=1-4$, together with the sum of the excited mode power (cf. Fig. 4a of Stavenga 2004). b Average fraction of the light power propagating inside the rhabdom boundary in the distal compartment from depth 0 to $20 \mu \mathrm{m}$ (dist) and from depth 220 to $280 \mu \mathrm{m}$ (prox)

accessible for absorption by the red screening pigments that surround the rhabdom. For each mode $p, \eta_{p}$ depends on the wavelength and the rhabdom diameter, which changes with the depth, $z$. The rhabdom of $P$. rapae crucivora tapers from a diameter of about $2.0 \mu \mathrm{m}$ distally to a diameter $1.5 \mu \mathrm{m}$ proximally, and hence the change of $\eta_{p}(\lambda)$ along the rhabdom has to be taken into account. Figure $5 \mathrm{~b}$ shows as an example the average values, $\bar{\eta}_{p}(\lambda)$, for a distal (depth $0-20 \mu \mathrm{m}$ ) and proximal (depth 220-280 $\mu \mathrm{m}$ ) compartment. Clearly, the light fraction inside the rhabdom boundary progressively diminishes with increasing wavelength and depth, especially for the higher order modes $(p>1)$. The waveguide effects therefore will distinctly modify the effective absorbance spectrum of the screening pigments.

In the calculations with the wave-optical model we used the excited mode powers of Fig. 5a and took into account the mode light fractions propagating inside the rhabdom boundary like those shown in Fig. 5b. Furthermore, we used the same absorbance spectra of the visual and screening pigments and the same tapetal reflectance spectra as before (Fig. 3a, b); the only modification was a value $0.14 \mu \mathrm{m}^{-1}$ taken for the peak absorbance coefficients of both the pale-red and deep-red screening pigments. The absorption spectra calculated for the different photoreceptors (Fig. 6a, b) appeared to be similar to the absorptance spectra calculated with the simple model (Fig. 3e, f). (The absorption has the dimension $W$, because the wavelengthindependent unit irradiance was conveniently chosen to be $1 \mathrm{~W} \mathrm{sr}^{-1} \mu \mathrm{m}^{-2}$; the dimension of the absorption becomes photons $\mathrm{s}^{-1}$ if the irradiance is 1 photon $\mathrm{s}^{-1} \mathrm{sr}^{-1} \mu \mathrm{m}^{-2}$.)

Figure $6 \mathrm{c}-\mathrm{f}$ presents the normalized absorbed light spectra, i.e., the calculated sensitivity spectrum, together with the corresponding experimentally determined sensitivity spectra. However, in the final calculations we have only taken into account the absorbed light from modes 1 and 2, because in the ultraviolet, at wavelengths $<400 \mathrm{~nm}$ where only modes 3 and 4 exist (Fig. 5), all photoreceptors showed distinct deviations between the calculated and experimental spectra. Using only modes 1 and 2, that is, neglecting modes 3 and 4, largely resolved the deviations.

A comparison of Fig. 6e, f with Fig. 3g, h clearly shows an improved correspondence of the calculated with the measured spectra, particularly for the PR receptors in type I and III ommatidia and also for the DR receptors in type IIf and IIm ommatidia. Notably the hump around $480 \mathrm{~nm}$ of the DR receptors, which was absent in the calculated spectra with the simple model, emerged with the waveoptics model. Some deviations remain, however, which we will discuss below.

\section{Discussion}

Modeling sensitivity spectra of Pieris rapae crucivora

The spectral sensitivities measured in photoreceptors of the Small White butterfly (Fig. 1) can already be quite well understood with a simple optical model (Fig. 3g, h); only for a few details a wave-optical treatment appears to be necessary (Fig. 6). It appears that several components of the butterfly ommatidium together determine the spectral sensitivity of the photoreceptors. The dominant component is the visual pigment, the rhodopsin, contained in the rhabdomeric microvilli. The spectra of the four visual pigments involved were derived from templates, using as peak wavelength $350,420,450$, and $560 \mathrm{~nm}$. A peak absorbance coefficient $\kappa_{\mathrm{v}, \max }=0.005 \mu^{-1}$ was assumed for all visual pigments, but variation of this value by $50 \%$ had very minor effects on the final sensitivity spectra. Of 
Fig. 6 Absorption spectra and photoreceptor spectral sensitivities calculated with a wave-optics model for the ommatidial types of Fig. 3. The absorption spectra $(\mathbf{a}, \mathbf{b})$ were calculated using the excited mode power of Fig. 5a and the fraction of the mode power propagating inside the rhabdom of Fig. 5b; however, the modes $p=3$ and $p=4$ were neglected. The visual and screening pigment absorbance spectra and tapetal reflectance spectra were the same as used in the calculations for Fig. 3 with the simple model.

Normalization of the absorption spectra yielded the spectral sensitivities of the

photoreceptors in ommatidia I (c), IIf (d), III (e), and IIm (f)
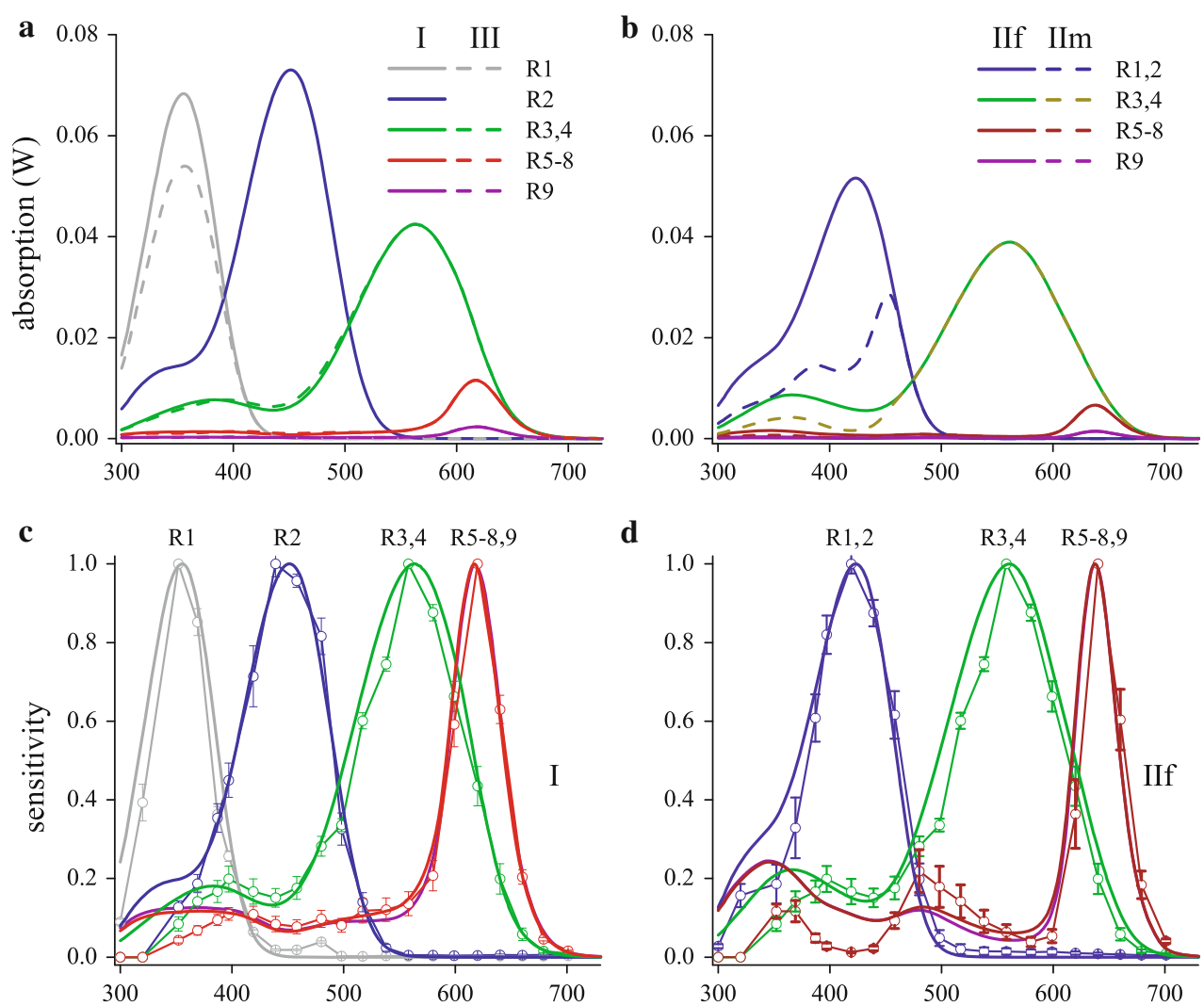

d
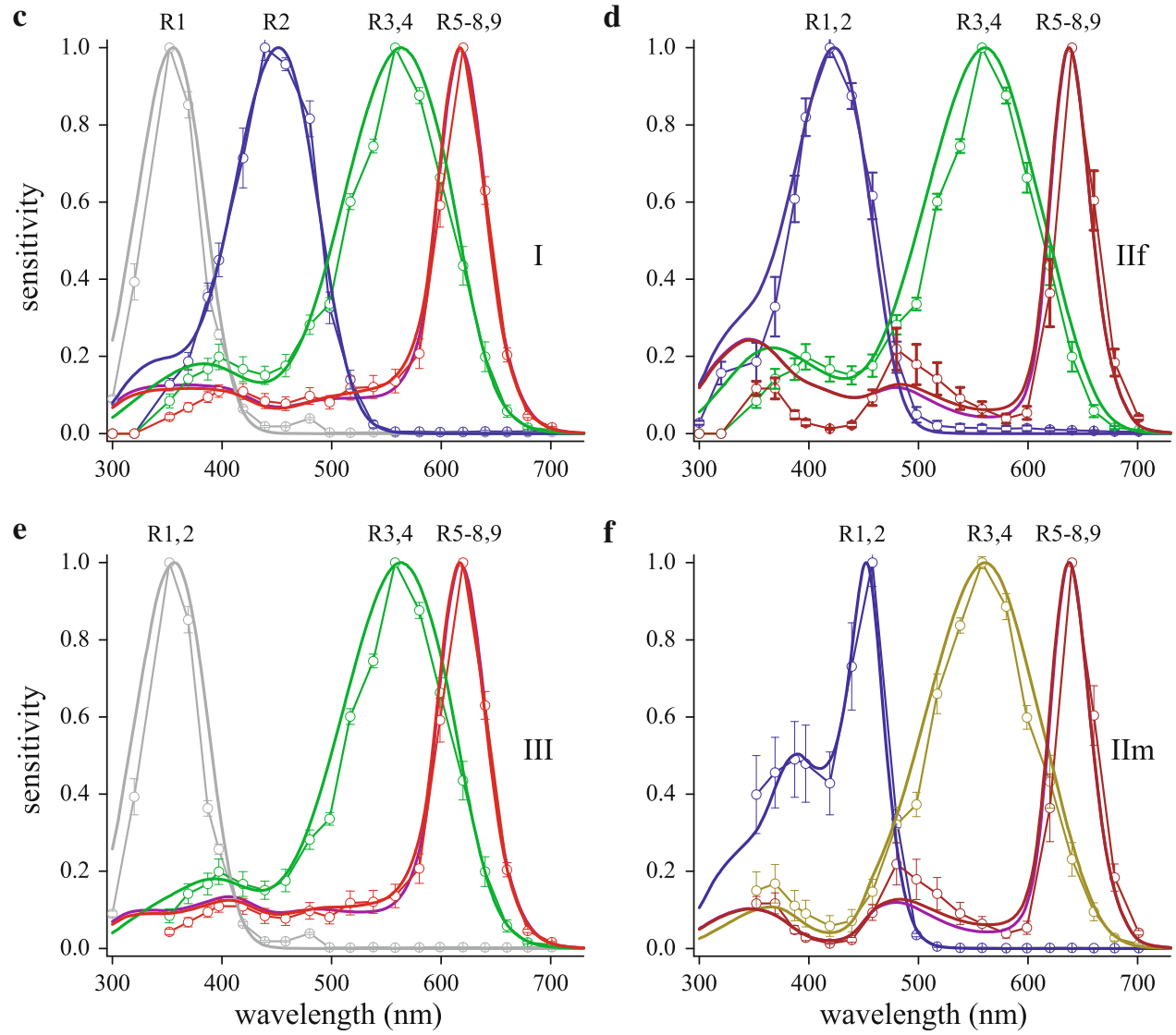

f

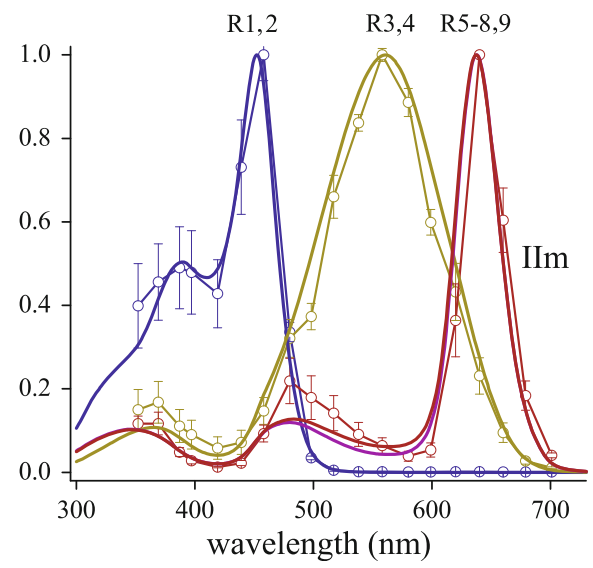

course, the photoreceptor absorptance values then change proportionally.

The sensitivity spectra of the R1-4 photoreceptors in the distal layer of type I, IIf and III ommatidia, calculated with either the simple or the wave-optical model, all resemble the template spectra, and the only slight deviations are due to some minor waveguide effects and lateral filtering. However, a pronounced filter effect is seen in the R1,2 photoreceptors of the IIm ommatidia, where the distally located fluorescent pigment, acting as a violet-absorbing filter, modifies the $\mathrm{V}$ receptor into a $\mathrm{dB}$ receptor. The additional effect of the violet filter is observable in the calculated R3,4 spectra of the IIm ommatidia as a deep trough in the violet wavelength range, corresponding to the trough in the spectra of the dG receptors (Fig. 1a). The latter spectral sensitivities were indeed obtained from R3,4 photoreceptors located in IIm ommatidia (Qiu and Arikawa 2003b).

The clusters of red pigment that surround the rhabdoms act as severe red filters, especially for the proximally located photoreceptors. With appropriately adjusted peak absorbance coefficients for the red pigments, implemented in the simple model, sensitivity spectra for the R5-8 receptors are obtained that match the sensitivity peaks of the PR (Fig. 3g) and DR (Fig. 3h) receptors. However, the calculated R5-8 sensitivity at wavelengths $\lambda<600 \mathrm{~nm}$ 
then is negligible, at variance with the experimental spectra (Fig. 1b). The wave-optics model yields much better results, and indeed spectra that match the experimental spectra over virtually the full wavelength range can be obtained, at least for the R5-8 in type I and III ommatidia. The calculated sensitivity spectrum of the DR receptors, R5-8 in type II ommatidia, differs between IIf and IIm ommatidia. Both show a hump around $480 \mathrm{~nm}$, similar to that in the measured spectra, but the calculated R5-8 spectra of type IIm ommatidia feature a distinct trough, due to the violet filter distally in the rhabdom. Only a very shallow dip is seen in the corresponding spectra of the R5-8 in type IIf ommatidia. The latter is caused by the R420 rhodopsin in the R1,2 photoreceptors of the distal rhabdom layer acting as a modest filter. The negligible sensitivity of the R5-8 photoreceptors at wavelengths $<580 \mathrm{~nm}$ resulting with the simple model versus the distinct sensitivity with an even pronounced hump emerging with the wave-optics model indicates the important influence of waveguide properties of the rhabdom on the photoreceptors' spectral characteristics. The fact that light propagates partly outside the rhabdom waveguide allows screening pigment located in the photoreceptor soma, adjacent to the rhabdom boundary, to act as a spectral filter. However, the pigment's effective absorbance spectrum is distinctly modified by the wavelength dependence of the light fraction propagating outside the rhabdom. That fraction increases strongly with increasing wavelength, and therefore absorption at the longer wavelengths is more strongly weighted than the shorter wavelength absorption. Thus, the effective absorbance spectrum of the red pigment in the short wavelength range is much suppressed relative to the longer wavelength part. The identical waveguide effect has been amply described before for the case of the pupil mechanism of blowfly photoreceptors (see Fig. 7 of Stavenga 2004).

The deviations between the calculated and experimental sensitivity spectra remaining with the wave-optical model, even after removing the contributions of modes 3 and 4 , may be easily attributed to the input parameters used in the model. For instance, a larger light flux in mode 2 relative to mode 1 will yield an enhanced hump around $480 \mathrm{~nm}$ in the sensitivity spectrum of the DR receptors. Furthermore, the rhabdoms are assumed to be circular cylinders, but their cross-sections are rather trapezoidal (type I), square (type II), and rectangular (type III) (see Fig. 6 of Qiu et al. 2002), and thus the light fractions $\eta_{p}(\lambda)$ of the propagating modes will differ from the values used. The assumption of a linear tapering rhabdom may also be slightly in error. Nevertheless, the spectral matches obtained suggest that the models are useful for a quantitative understanding of the Small White's sensitivity spectra.
The calculations show that at wavelengths $<600 \mathrm{~nm}$ the light flux arriving at the basal level of the rhabdom is negligible. This underscores previous assumptions that the basal R9 photoreceptors contain the long-wavelength absorbing rhodopsin, R560. Indeed, the only sensitivity spectrum reported for an $\mathrm{R} 9$ photoreceptor of $P$. rapae crucivora (Shimohigashi and Tominaga 1991) is very similar to the red-peaking sensitivity spectra recorded from R5-8 photoreceptors in type I and III ommatidia (Fig. 1). Of course, the absorptance of all R9s is distinctly lower than that of the R5-8s (and certainly much lower than that of R3,4; Figs. 3e, f, 6a, b), but the sensitivity is not at all negligible. Normalization shows that in the different ommatidial types the sensitivity spectra of the R5-8 and the R9 are virtually identical. Notwithstanding their low sensitivity, the R9 photoreceptors presumably have a role in butterfly vision, but what its function can be is unknown.

\section{The tapetum}

A persistent question in the study of butterfly vision has been the function of the tracheolar tapetum. It acts as a reflector and thus it is expected to enhance the sensitivity of some photoreceptors. The tapeta can potentially only have this effect at those wavelengths where the light reaching the tapetum is non-negligible, i.e., at $\lambda>600 \mathrm{~nm}$ (Fig. 3c, $\mathrm{d} ; 400 r$ ). The long-wavelength part of the tapetal reflectance spectrum was derived from the calculations using the average of measured eye shine spectra (Qiu et al. 2002). The eye shine spectrum is not at all identical for all ommatidia. Both the left and right ends of the spectra vary (Qiu et al. 2002), indicating that both the tapetal reflectance spectrum and the effective absorbance spectrum of the red screening pigments vary among the ommatidia. This partly explains the variability of the experimental sensitivity spectra of the R5-8 photoreceptors. When using the calculated tapetal reflectance spectra, where the value in the wavelength range below $\sim 600 \mathrm{~nm}$ was given the maximal possible value, 1 , it appears that only the basal photoreceptor, R9, may gain a little sensitivity, at most $50 \%$. If the maximal reflectance of the tapetum is much lower than the assumed 100\%, which is more than likely (Nilsson and Howard 1989), this sensitivity enhancement shrinks accordingly. The additional light absorbed from the flux at the reverse pathway by $\mathrm{R} 5-8$ photoreceptors is anyhow extremely minor. Thus, it seems that the function of the tapetum for enhancing light sensitivity is at most moderate, at least in $P$. rapae crucivora.

Interestingly, members of the pierid genus Anthocharis have lost the tracheolar tapetum and thus their eyes exhibit no eye shine (Takemura et al. 2007). The tapetum is also absent in all papilionids. Most butterfly eyes nevertheless have a very well developed tapetum and a vivid eye shine 
(Miller and Bernard 1968; Stavenga 2002). It remains to be seen whether in those cases the tapeta do have a distinct visual function.

Visual sensitivity and natural illuminants

The peak absorptance of the individual photoreceptors of $P$. rapae crucivora decreases with increasing peak wavelength (Fig. 3e,f), which was calculated using the assumption that the peak absorbance coefficient of all rhodopsins involved is identical. The latter may be questionable, since it is rather the integral of the spectrum (the oscillator strength) that may be constant. The ratio of the integrals of the rhodopsin spectra when taking a constant peak absorbance coefficient is R350:R420:R450:R560 = 1:1.3:1.5:1.9. Consequently, when the integral of the spectrum is constant instead of the peak absorbance coefficient, the decrease of the photoreceptor absorptance peaks shown in Fig. 3e, $\mathrm{f}$ becomes even stronger, proportionally with these ratio values.

Another complication is the implicit assumption that all photoreceptors have the same rhodopsin concentration, which will not be the case in most natural situations. We recall that the rhodopsin molecules of butterflies, like those of other invertebrates, upon absorption of a photon convert into a thermostable metarhodopsin state, which then in turn can be photoreconverted into the native rhodopsin state. Continuous illumination establishes a photoequilibrium with a rhodopsin-metarhodopsin ratio depending on the spectral properties of the two visual pigment states and the spectral composition of the illuminant. The present calculations then clearly will no longer hold. For instance, the metarhodopsins present in the rhabdom will then act as an additional spectral filter and thus modify the spectral sensitivity of the colocalized photoreceptors. An even more complicated aspect is the visual pigment content under natural, daylight conditions. In the dominant population of photoreceptors with green rhodopsins illumination with prolonged broad-band, bright natural light creates a high metarhodopsin concentration, which is degraded, leading to visual pigment loss (see Stavenga and Hardie 2010). Preliminary calculations indicate, however, that the filtering effects will be mostly minor, and especially due to the normalization procedures, the spectral sensitivities will not significantly differ from those calculated above.

The same holds for another important parameter which has been neglected in the calculations, the polarization sensitivity of the photoreceptors. Anatomical studies have demonstrated clear differences in the microvillar orientations of the different photoreceptors in the three ommatidial types (Qiu et al. 2002), which will result in different polarization sensitivities. This will probably also have minor consequences for the absorptances and the spectral sensitivities.
Function of the different sets of photoreceptors of Pieris rapae crucivora

The compound eyes of $P$. rapae crucivora are marked by a diversified set of photoreceptors, especially in the violetblue wavelength range. This diversification has probably arisen because of fine discrimination of interesting spectral objects reflecting at those wavelengths. Accordingly, we put forward the hypothesis that the diversification of shortwavelength sensitive photoreceptors occurred for improving the discrimination of the colours of conspecifics (Arikawa et al. 2005; Stavenga and Arikawa 2006). The wings of male $P$. rapae crucivora are dorsally mostly white due to strongly scattering granules that fill the wing scales. The granules contain the UV-absorbing pigment leucopterin, and the wings therefore reflect little in the ultraviolet (Fig. 7a, b). The undersides of the hindwings are somewhat yellow because the granules of part of the wing scales contain the UV- and blue-absorbing xanthopterin (Fig. 7c, d). Female $P$. rapae crucivora wings contain few granules, but those present have either leucopterin and xanthopterin, resulting in an overall weakly white colouration (Fig. 7c-h). Measured reflectance spectra (Fig. 7i,j) reveal the contributions of the different pterins (Stavenga et al. 2006; Wijnen et al. 2007; Giraldo and Stavenga 2007).

To investigate the visibility of the wings we have multiplied the average of the spectra of the white wing parts of the male (spectra \#1-3 of Fig. 7i) with a D65 daylight spectrum converted into photons, and then normalized the resulting spectrum (bold dark-blue curve in Fig. 8). We repeated the same procedure with the reflectance spectrum of the under side of the male hindwing (bold yellow curve in Fig. 8). In addition, Fig. 8 presents the sensitivity spectra of all photoreceptor classes (except the R9s) obtained with the wave-optical model. The spectral sensitivities of the male's $\mathrm{dB}$ receptor and the female's $\mathrm{V}$ receptor as well as the green $(\mathrm{G}$ and $\mathrm{dG})$ receptors are coloured, but the other spectra are presented as black curves. Figure 8 shows that the two wing reflection spectra have little overlap with the spectra of the UV receptor, but the wing spectra almost fully overlap both $\mathrm{G}$ and $\mathrm{dG}$ (and both PR and DR) receptors. Discrimination of the wing spectra by only the UV-and G-receptors will be difficult. Inclusion of the blue (B) receptor will improve the discrimination of the wing spectra considerably because it overlaps much more with the white wing reflection spectrum than with the yellow wing spectrum. The overlap of the wing spectra with the sensitivity spectra of the $\mathrm{V}$ and $\mathrm{dB}$ receptors is also distinctly different, and therefore the neural processing of the colour discrimination with the more narrow-band spectrum of the male's $\mathrm{dB}$ receptors is possibly more acute than that with the female's $\mathrm{V}$ receptors. At least for the discrimination of the reflection spectra 
Fig. 7 UV and RGB photographs of the upper (dorsal) and lower (ventral) side of the wings of male (a-d) and female (e-h) Pieris rapae crucivora and their reflectance spectra. The numbers in the wing photographs $(\mathbf{a}-\mathbf{h})$ correspond to the numbers of the spectra $(\mathbf{i}, \mathbf{j})$
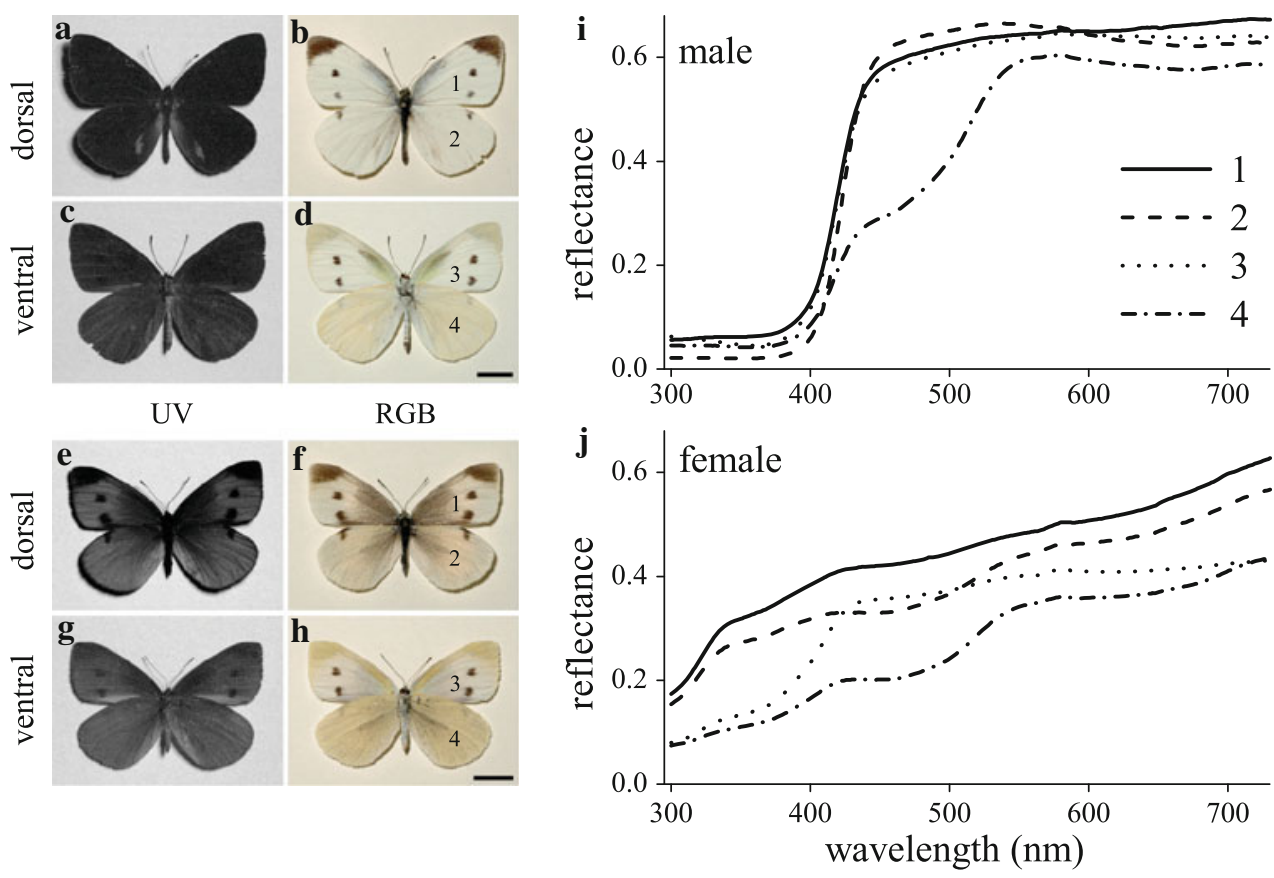

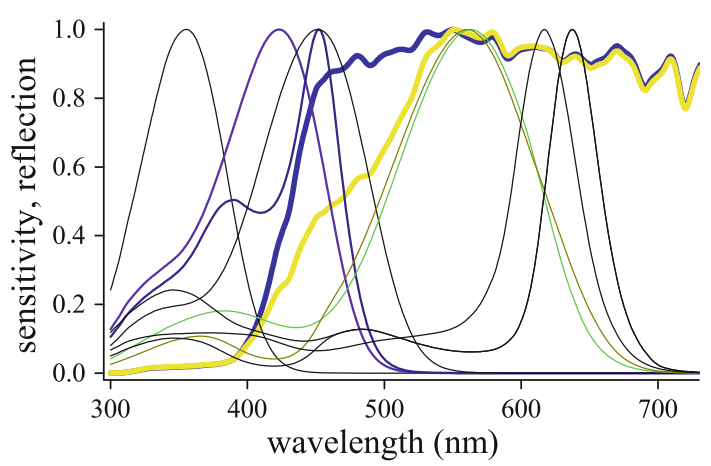

Fig. 8 Photoreceptor spectral sensitivities and reflection spectra of male wings. The reflectance spectra of the male dorsal wing (averaged of spectra 1-3 together with spectrum 4 of Fig. 7i) were multiplied with a D65 environmental light spectrum and subsequently normalized (bold blue and yellow spectra). The spectral sensitivities of Fig. 6 are presented as thin curves. The $\mathrm{V}$ receptor of ommatidial type IIf and the $\mathrm{dB}$ receptor of type IIm are shown in violet and blue, and the $\mathrm{G}$ and $\mathrm{dG}$ receptors are shown as green and olive-coloured (the spectra of the UV, PR and DR receptors are given in black)

of the female, we have to conclude that the sensitivity spectrum of the $\mathrm{dB}$ receptor has little advantages over that of the V-receptor, because of the wings' very shallow, broad-ranged spectra.

Although the functional significance of the variety of B receptor classes is not yet clear at the present stage, the diversification of these receptors in P. rapae crucivora is accompanied by $\mathrm{B}$ opsin duplication (Wakakuwa et al. 2010), which is shared by other species in the family Pieridae (Awata et al. 2009). Comparative analyses among pierid species would probably provide clues to understand the biological function of the $\mathrm{B}$ receptor diversification.
Acknowledgments Dr Jeremy Niven critically read the manuscript. Hein Leertouwer made the photographs of Fig. 7. This work was supported by AFOSR/EOARD grant no. FA8655-08-1-3012 (to D.G.S.), and by the JSPS grant no. 21247009 and MAFF grant no. INSECT-1101 (to K.A.).

Open Access This article is distributed under the terms of the Creative Commons Attribution Noncommercial License which permits any noncommercial use, distribution, and reproduction in any medium, provided the original author(s) and source are credited.

\section{References}

Arikawa K, Stavenga DG (1997) Random array of colour filters in the eyes of butterflies. J Exp Biol 200:2501-2506

Arikawa K, Wakakuwa M, Qiu X, Kurasawa M, Stavenga DG (2005) Sexual dimorphism of short-wavelength photoreceptors in the Small White butterfly, Pieris rapae crucivora. J Neurosci 25:5935-5942

Awata H, Wakakuwa M, Arikawa K (2009) Evolution of color vision in pierid butterflies: blue opsin duplication, ommatidial heterogeneity and eye regionalisation in Colias erate. J Comp Physiol A 195:401-408

Bernhard CG, Miller WH, Møller AR (1965) The insect corneal nipple array. Acta Physiol Scand 63(Suppl 243):1-25

Giraldo MA, Stavenga DG (2007) Sexual dichroism and pigment localization in the wing scales of Pieris rapae butterflies. Proc R Soc B 274:97-102

Govardovskii VI, Fyhrquist N, Reuter T, Kuzmin DG, Donner K (2000) In search of the visual pigment template. Vis Neurosci 17:509-528

Miller WH, Bernard GD (1968) Butterfly glow. J Ultrastruct Res 24:286-294

Nilsson D-E, Howard J (1989) Intensity and polarization of the eyeshine in butterflies. J Comp Physiol A 166:51-56

Nilsson D-E, Land MF, Howard J (1988) Optics of the butterfly eye. J Comp Physiol A 162:341-366 
Qiu X, Arikawa K (2003a) Polymorphism of red receptors: sensitivity spectra of proximal photoreceptors in the small white butterfly Pieris rapae crucivora. J Exp Biol 206:2787-2793

Qiu X, Arikawa K (2003b) The photoreceptor localization confirms the spectral heterogeneity of ommatidia in the male small white butterfly, Pieris rapae crucivora. J Comp Physiol A 189:81-88

Qiu X, Vanhoutte KJA, Stavenga DG, Arikawa K (2002) Ommatidial heterogeneity in the compound eye of the male small white, Pieris rapae crucivora. Cell Tissue Res 307:371-379

Shimohigashi M, Tominaga Y (1991) Identification of UV, green and red receptors, and their projection to lamina in the cabbage butterfly, Pieris rapae. Cell Tissue Res 247:49-59

Stavenga DG (2002) Colour in the eyes of insects. J Comp Physiol A 188:337-348

Stavenga DG (2003) Angular and spectral sensitivity of fly photoreceptors. II. Dependence on facet lens F-number and rhabdomere type in Drosophila. J Comp Physiol A 189:189-202

Stavenga DG (2004) Angular and spectral sensitivity of fly photoreceptors. III. Dependence on the pupil mechanism in the blowfly Calliphora. J Comp Physiol A 190:115-129

Stavenga DG (2010) On visual pigment templates and the spectral shape of invertebrate rhodopsins and metarhodopsins. J Comp Physiol A 196:869-878

Stavenga DG, Arikawa K (2006) Evolution of color and vision of butterflies. Arthropod Struct Dev 35:307-318

Stavenga DG, Hardie RC (2010) Metarhodopsin control by arrestin, light-filtering screening pigments, and visual pigment turnover in invertebrate photoreceptors. J Comp Physiol A. doi:10.1007/ s00359-010-0604-7

Stavenga DG, Smits RP, Hoenders BJ (1993) Simple exponential functions describing the absorbance bands of visual pigment spectra. Vis Res 33:1011-1017

Stavenga DG, Giraldo MA, Hoenders BJ (2006) Reflectance and transmittance of light scattering scales stacked on the wings of pierid butterflies. Opt Express 14:4880-4890

Takemura SY, Stavenga DG, Arikawa K (2007) Absence of eye shine and tapetum in the heterogeneous eye of Anthocharis butterflies (Pieridae). J Exp Biol 210:3075-3081

van Hateren JH, Nilsson D-E (1987) Butterfly optics exceed the theoretical limits of conventional apposition eyes. Biol Cybern 57:159-168

Wakakuwa M, Stavenga DG, Kurasawa M, Arikawa K (2004) A unique visual pigment expressed in green, red, and deep-red receptors in the eye of the small white butterfly, Pieris rapae crucivora. J Exp Biol 207:2803-2810

Wakakuwa M, Stavenga DG, Arikawa K (2007) Spectral organization of ommatidia in flower-visiting insects. Photochem Photobiol 83:27-34

Wakakuwa M, Terakita A, Koyanagi M, Stavenga DG, Shichida Y, Arikawa K (2010) Evolution and mechanism of spectral tuning of blue-absorbing visual pigments in butterflies. PLoS ONE 5:e15015

Wijnen B, Leertouwer HL, Stavenga DG (2007) Colors and pterin pigmentation of pierid butterfly wings. J Insect Physiol 53:12061217 